\title{
A Qualitative Case Study Exploring Hand-Hygiene Standards in an Intensive Care Unit
}

\author{
Lyndon Augustine \\ Capitol Technology University \\ Walter McCollum \\ Walden University \\ Richard Brown \\ Capitol Technology University \\ Phoenix Mourning-Star \\ The George Washington University
}

\begin{abstract}
Healthcare-associated infections (HAIs) are infections that occur in patients during their time of care in a hospital. Considerable emphasis is currently placed on reducing HAIs through improving hand-hygiene $(\mathrm{HH})$ compliance among healthcare professionals because HAIs are a critical challenge to public health in the United States. By focusing on meeting Centers for Disease Control and Prevention HH standards, the purpose of this qualitative research was to explore how noncompliance with these standards and lack of technology usage affect HAIs in the intensive care unit. Additionally, the goal of this research was to explore behavioral factors and best practices that influence compliance rates in intensive care units. Thereafter, the researcher provided recommendations for healthcare leadership to address the phenomenon of HAIs.
\end{abstract}

Keywords: hand hygiene, healthcare-associated infections, hand-hygiene compliance, healthcare worker

\section{Introduction}

Healthcare-associated infections (HAIs), also referred to as nosocomial infections, are those that occur in patients during their time of care in a hospital. HAIs can affect a patient in any type of setting where they receive care and can even appear up to $48 \mathrm{hr}$ after the patient has been discharged (Baslyman et al., 2015). HAIs represents the most frequent adverse event occurring in healthcare in which one in every 10-20 patients in the United States is subjected to exposure (Radhakrishna et al., 2015). According to Radhakrishna et al., contaminated hands of healthcare

The doctoral research is a collaborated accomplishment with everyone who supported my academic goals. This was a life changing journey of investigative research and scholarly writing to reflect my understanding and analytical rigor of my research. The first author thank my chair, Dr. Walter McCollum, for his exceptional guidance, constructive feedback, and unwavering commitment towards the assurance of a purposeful study. I appreciate his doctoral best practices and guidance that was provided to help me attain my academic goals. The first author especially thank the executive management team at the university hospital for approving my study. Lastly, the first author would like to thank Dr. Richard Brown and Dr. Phoenix Mourning-Star for their efforts as doctoral subject matter experts throughout this rigorous process. 
workers (HCWs) can increase the risk of patients developing HAIs. Furthermore, evidence-based guidelines have emphasized that the underlying reason for cross-transmission of infections in any healthcare setting was poor hand-hygiene $(\mathrm{HH})$ compliance by HCWs.

HH compliance is defined as properly washing one's hands with soap and water or an antiseptic agent before and after all patient or patient-environment contact (Hong et al., 2015). As a result, proper $\mathrm{HH}$ compliance is one of the most effective practices that HCWs can perform to reduce the risk of HAIs in a hospital (McGuckin \& Govednik, 2015). Despite this simple and inexpensive prevention measure, as stated by Marques et al. (2017), proper HH compliance continuously remains low in hospitals. For example, compliance levels for HCWs remain at or below $50 \%$ in the United States (McGuckin \& Govednik, 2015). Furthermore, the World Health Organization (WHO, 2011) reported that out of all the care units within a hospital, the risk of acquiring a HAI is significantly higher in intensive care units (ICUs), which usually have low HH compliance levels.

Considerable emphasis is currently placed on reducing HAIs through improving HH compliance among healthcare professionals because HAIs are a critical challenge to public health in the United States. For example, more than 98,000 deaths per year in the United States have been attributed to HAIs (Hong et al., 2015). Additionally, these infections have contributed to more deaths than AIDS, breasts cancer, and car accidents combined (Marques et al., 2017). As a result, healthcare leaders must seek out strategies to improve $\mathrm{HH}$ compliance as a means to reduce HAIs.

In addition to the social impact, HAIs have also placed a financial burden on the U.S. healthcare system. For instance, McGuckin and Govednik (2015) purported that HAIs in U.S. acute care hospitals have led to a burden of $\$ 96-\$ 147$ billion annually on the U.S. health system and have affected one in 20 hospital patients. In the 2013 Centers for Disease Control and Prevention (CDC, 2013) report on antibiotic resistance threats, it was estimated that the direct healthcare cost of antibiotic resistance was near $\$ 20$ billion annually. However, this figure does not include the additional estimated cost of $\$ 35$ billion to society, which was attributed to losses in productivity. Despite this, there have been no lasting strategies implemented to reduce these costs.

Hence, healthcare leaders will need to take an active role toward motivating and changing HCWs behaviors to comply with the various practices used to help the HCW meet $\mathrm{HH}$ compliance. The practice of choice used by healthcare institutions to help HCWs meet HH compliance varies. However, the three main practices used to meet $\mathrm{HH}$ compliance are direct observation, product volume measurement, and electronic compliance monitoring (ECM) systems (Hagel et al., 2015). Nevertheless, of the three practices cited, direct observation is the most commonly used and is known as the gold standard.

\section{Statement of the Problem}

Hospitals and infection prevention specialists have attempted to achieve high levels of compliance with $\mathrm{HH}$ protocols for many decades. Even though the gold standard that is used to monitor $\mathrm{HH}$ compliance is direct observation, the effectiveness of this practice is under scrutiny to mitigate the risks of spreading HAIs (Baslyman, 2015). This problem of spreading HAIs can create an unstable environment for patients in that it affects patient care. Consequently, Hong et al. (2015) noted that healthcare organizations' noncompliance with CDC HH standards poses the risk of increasing HAIs due to insufficient technology usage.

\section{Purpose of the Study}

The purpose of this qualitative research was to explore how noncompliance with CDC HH standards and lack of technology usage impacts HAIs in the ICU. Additionally, the goal of this research was to 
explore behavioral factors and best practices that influence compliance rates in ICUs. The research questions (RQs) were as follows:

$R Q$ 1: What are the best practices and behavioral factors that affect $\mathrm{HH}$ standards and compliance rates in ICUs?

$R Q$ 2: How can leadership motivate HCWs in ICUs to meet CDC HH standards and compliance requirements?

$R Q$ 3: How does technology impact HH compliance rates in ICUs to inform decision-making?

The significance of this research was that it provided a different perspective on the already existing problem in healthcare as it relates to HCWs not maintaining $\mathrm{HH}$ compliancy. Through existing scholarly literature and subject matter expert engagements in the field, the researcher added depth and situational awareness to the impacts of best practices and HCW behavior, leaderships' ability to motivate HCWs to meet HH standards, and current technology offerings to meet HH compliance rates. From a beneficial perspective, this research may benefit patients to help reduce length of stay in hospitals, it may benefit technology companies to help identify barriers toward integrating technologies within healthcare, and it may benefit healthcare leadership to identify any gaps between HCW behavior and organization culture. Finally, this research may help healthcare organizations understand the impact that technology can have as a solution to inform decisionmaking because, currently, radio frequency identification (RFID) technologies are underused within healthcare institutions to help meet HH compliance (Carr, 2015).

\section{Definition of Terms}

This research focused on the following concepts:

Catheter-associated urinary tract infection (CAUTI): an infection attributed to the use of an indwelling urinary catheter at the time of, or within 48 hours before, the development of a UTI (CDC, 2017a)

CDC: a government agency that serves to protect the public health and safety by providing information to the citizens of the United States (CDC, 2017b)

Central-line-associated bloodstream infection (CLABSI): healthcare-associated bloodstream infection in a patient who had an intravascular catheter (e.g., central line) at the time of, or within $48 \mathrm{hr}$ before, the development of a bloodstream infection (CDC, 2017a)

Clostridium difficile: a bacterium that causes an inflammation of the colon (called colitis); diarrhea and fever are the most common symptoms (CDC, 2017c)

$\mathrm{HH}$ : washing hands with alcohol rub or with traditional soap and water before and after every contact (CDC, 2017c)

$\mathrm{HH}$ compliance rate: the number of correct $\mathrm{HH}$ opportunities divided by the total number of HH opportunities (Van Dijk et al., 2019)

HCWs: doctors, registered nurses, and technicians involved in direct patient care (Van Dijk et al., 2019)

Methicillin-resistant Staphylococcus aureus: infection caused by methicillin-resistant bacteria; responsible for many infections resulting in increased hospitalization and sometimes death (CDC, 2017c)

RFID: uses radio waves to automatically detect objects (Carr, 2015)

Ventilator-associated pneumonia (VAP): a healthcare-associated pneumonia that occurs in a patient who was intubated and ventilated to assist or control respiration continuously at the time of, or within $48 \mathrm{hr}$ before, the onset of the pneumonia (CDC, 2017c) 


\section{Assumptions and Limitations}

The first presumed assumption was that the secondary data collected would be cost effective and convenient. A second assumption around the secondary data was that it would be believable and trustworthy because this is the same data that is provided to the CDC. In addition, because triangulation was employed, the assumption was that any form of biases that the researcher may have had toward the research would not interfere with the data collection and analysis. Accordingly, through multiple data collection methods, this would provide a more in-depth clarification into the study.

In terms of limitations, the first was that the researcher was not able to determine if the responses from the questionnaire were truthful or not. Secondly, for the secondary data, the researcher did not participate in the data collection process and thus was not able to determine whether the Hawthorne effect compromised the integrity of the data because various clinical professionals collected the data, which were then loaded into the hospitals' HH survey portals. The final limitation of the study was that it was limited to an ICU within a private teaching hospital in the Washington, DC, area, so the results may not be transferable to another region of the United States.

\section{Literature Review}

As noted by Simon and Goes (2013), a thorough, sophisticated, and extensive literature review is the foundation and inspiration for substantial and contributory research. For this reason, the literature review focused on the various HH monitoring methods, current compliant levels, common HAIs, contributing factors for noncompliance, RFID technology to monitor HH compliance, and critical success factors and barriers toward adopting technological $\mathrm{HH}$ solutions.

\section{Best Practices for Monitoring HH Compliance}

As highlighted by Gould et al. (2017), the purpose of HH is to break the chain of HAIs. The first recommended practice is direct observation, which has been described as the gold standard approach to $\mathrm{HH}$ audit. This approach is favored by WHO because, at the time the guidelines were published, it was the only method described that could detect all HH opportunities (Gould et al., 2017). The second recommended practice is product volume measurement. This practice records each time an alcohol-based hand rub (or soap) dispenser has been accessed (electronic dispenser counters), which can provide information regarding the frequency of $\mathrm{HH}$ events (Boyce, 2017). The third recommended practice, noted by Ellingson et al. (2014), is self-reporting, which can raise an individual's awareness of $\mathrm{HH}$ practice. The final recommended practice is using ECM systems, which fall into three major categories: activity monitoring systems, systems that include the wearing of RFID electronic badges by the HCW, and camera-based systems (Boyce, 2017).

\section{Current Compliance Levels With CDC HH Standards}

In a systematic review of 96 studies from around the world, Erasmus et al. (2010) reported a median $\mathrm{HH}$ compliance rate of only $40 \%$ in hospital units of all kinds. In another review from Neo, SaghaZadeh, Vielemeyer, and Franklin (2016), HH compliance only reached 30-40\% in ICUs. Regulatory expectation is that healthcare organizations must maintain a $90 \%$ record to be considered in compliance.

\section{Common HAls}

Many noted HAIs plague patients every year. Although this may be true, Klevens et al. (2007) reported that the four most common HAIs are CAUTI (32\%), surgical-site infections (22\%), VAP (15\%), and CLABSI (14\%). 


\section{Contributing Factors for Noncompliance}

Chassin, Mayer, and Nether (2015) presented factors on an individual level such as lack of accountability and the HCW being distracted or too busy. Nevertheless, Baker and Wilson (2007) cited that noncompliance with $\mathrm{HH}$ regulations is mostly related to excessive workloads, insufficient time, and staff shortages. Case in point, Martins Pereira et al. (2016) noted that compared to palliative care, working in ICUs more than doubled the likelihood of HCWs exhibiting burnout.

\section{RFID Technology to Monitor HH Compliance}

The findings for using RFID sensing technology were that there was a successful detection rate of $97.5 \%$ of the compliant events and $100 \%$ of the noncompliant events. Overall, the RFID sensing technology delivered an accuracy of $98.75 \%$ (Hong et al., 2015). In a similar study conducted by Radhakrishna et al. (2015), the findings for using RFID technology included that using automated systems increased sanitizer use among HCWs, which means that using RFID has the potential to change behavior.

\section{Critical Success Factors and Barriers Toward Adopting Technological HH Solutions}

Al Salman, Hani, de Marcellis-Warin, and Isa (2015) purported that resistance to change is often a significant concern in a healthcare environment when introducing a new technology and is difficult to overcome. With this in mind, in this qualitative study of 61 healthcare administrators' perspectives, Kash, Spaulding, Johnson, Gamm, and Hulefeld (2014) disclosed 10 key success factors related to the implementation of strategic change within hospitals and healthcare systems. The top were culture and values, business processes, and people and engagement. On the contrary, as Carr (2015) reported, major barriers to the adoption of RFID technology are the high cost, followed by security concerns, privacy, trust, and supplier technical support.

\section{Methodology}

\section{Research Method and Design}

The purpose of this qualitative research was to explore how noncompliance with CDC HH standards and lack of technology usage impacts HAIs in the ICU. Additionally, the goal of this research was to explore behavioral factors and best practices that influence compliance rates in ICUs. In terms of methodology, a qualitative method was chosen for various reasons. First of all, as noted by Creswell (2005), qualitative research can offer valuable insight on behavioral factors and is often the first step in developing a strategy. The second reason why a qualitative method was best suited for this research was that qualitative research is an approach for exploring and understanding the meaning of individuals or groups to a social or human problem (Creswell, 2014). In addition, Glogowska (2011) argued that researches use qualitative research to study and examine phenomena from a nonobject perspective. The above reasons aligned with the study selected because the goal was to understand behavioral factors through the research of participants' perspective.

In terms of design, a case study was chosen for the following reasons. First, as highlighted by Yin (2009), a case study design asks what and how questions, which aligns with the RQs for this research. Second, according to Yin, a case study design is usually the preferred method used in health and social sciences as an approach for examining complex issues. Yin also stated that a case study approach is best for exploring and describing a phenomenon in the form of real life. In summary, a qualitative case study was appropriate for this research because, as indicated by Yin, a qualitative case study design is the best selection for studying humans in a single organization, which in this research was a hospital. 


\section{Sampling Method}

The population for this study included one ICU in a private university hospital in Washington, DC. However, the purposeful sample was limited to doctors, nurses, and patient care technicians in the ICU. This sample population was appropriate for this case study because the identified participants were the ones that have $24-\mathrm{hr}$ access to the ICUs to provide care. The sample size for this research was $35 \mathrm{HCWs}$ (15 doctors, 15 nurses, and five patient care technicians) within the ICU. The sampling number was determined based on the amount of fulltime workers employed within the ICU.

\section{Instrumentation}

The notable sources to use for case study design can include documentation, archival records, interviews, direct observation, participant observation, and physical artifacts (Yin, 2009). With this in mind, the researcher used secondary data, direct observation, and a questionnaire as the instruments. However, as Creswell (2014) noted, in qualitative research, the researcher can also be included as an instrument because the researcher collects data themselves through examining documents, or observing behavior.

\section{Data Collection and Analysis}

The researcher incorporated Yin's (2009) approach by multiple sources of data collection (i.e. direct observation, questionnaires, and secondary data). Data for this research were collected from a pilot study of four participants and from the main study, which consisted of 3.5 years of secondary data from the organization, direct observation of 35 participants (15 doctors, 15 nurses, and five patient care technicians), and questionnaires from 21 participants (14 doctors, three nurses, and four patient care technicians). The data set from the secondary data consisted of historical data pertaining to compliance rates, types of HAIs acquired, quantity of HAIs accumulated for each year, and standardized infection ratio (SIR). For the direct observation, the researcher captured observational notes as HCWs entered and exited patient rooms and were labeled with an assigned physician, nurse, or patient care technician ID.

The questionnaire consisted of 16 open-ended questions, which provided an in-depth grasp on the level of awareness of $\mathrm{HH}$ practices and behavioral factors as it relates to CDC HH standards and compliance rates. What's more, the questionnaire gave insight into the three RQs. The identity of each participant was recorded and a code identifier was assigned. The 21 questionnaires were transcribed and organized in Microsoft Word by participant (three nurses, four patient care technicians, and 14 physicians) for importing into NVivo 12 qualitative software. Each line was manually read and coded to correspond to three parent nodes: best practices and behavioral factors, how leadership can motivate HCWs, and impact of technology. The data gathered from this coding process were then used to help identify any themes from the physicians, nurses, and patient care technicians.

\section{Credibility and Validity}

According to Elo et al. (2014), the demonstration of the trustworthiness of the data collected is one aspect that supports a researcher's ultimate argument. Therefore, to ensure trustworthiness, the researcher focused on four criteria associated with trustworthiness in qualitative research. The first criterion was confirmability, which refers to objectivity and implies that the data accurately represent the information that the participants provided and interpretations of the data are not invented by the inquirer (Elo et al., 2014). To establish this criterion, an audit trail was performed to keep a record of what was done in the investigation. The second criterion was dependability, which 
refers to the stability of data over time and under different conditions (Elo et al., 2014). To establish this criterion, an inquiry audit was conducted, which involved having a researcher not involved in the research process to examine both the process and product of the research study. The third criterion was transferability, which refers to the extent to which the findings can be transferred to other settings or groups (Elo et al., 2014). To establish this criterion, the researcher used thick description as a way of achieving a type of external validity. The fourth criterion was credibility, which refers to the verisimilitude and plausibility of the research findings (Pandey \& Patnaik, 2014). To establish this criterion, the researcher first practiced prolonged engagement. This involved spending adequate time observing various aspects of the setting, speaking with a range of people, and developing relationships and rapport with members of the organization. Secondly, triangulation was employed to establish credibility. As Pandey and Patnaik (2014) purported, triangulation involves using multiple data sources in an investigation to produce greater understanding.

\section{Findings}

\section{Evaluation of Research Data}

There were three RQs used in this study to help guide the methodology and research design. The researcher used all three data collection methods to address RQ1. The first data collection that was used to address RQ1 was the questionnaire. The response rate from the questionnaire was $60 \%$, in that 21 out of 35 were completed. The researcher compared the frequency of responses for 21 participants to identify any themes from the parent node, which were best practices and behavioral factors. The analysis of six questions was used in Table 1 to address RQ1.

Table 1. Questionnaire to Address Research Question 1: Best Practices and Behavioral Factors

\begin{tabular}{llcc}
\hline Survey Question & \multicolumn{1}{c}{ Top Response } & $\boldsymbol{n}$ & $\mathbf{\%}$ \\
\hline 1. Behavioral factors hinder not practicing HH & In a hurry-emergencies & 9 & $43 \%$ \\
& $\begin{array}{l}\text { Empty or malfunctioning } \\
\text { 2. Other factors hinder not practicing HH }\end{array}$ & 10 & $48 \%$ \\
& $\begin{array}{l}\text { dispensers } \\
\text { 3. Your role compliance requirements }\end{array}$ & 18 & $86 \%$ \\
4. View of current HH practice to monitor HH & Satisfactory & 9 & $43 \%$ \\
5. Other practices to monitor HH-favorite & Like-recommend-favor & 12 & $57 \%$ \\
6. What you do if team members not practicing & Remind & 17 & $81 \%$ \\
HH & & & \\
\hline
\end{tabular}

Note. $N=21 . \mathrm{HH}=$ hand hygiene.

\section{Secondary Data Results to Address RQ1 (Excluded From RQ2 and RQ3)}

In addition to using a questionnaire to address RQ1, the researcher also used the secondary data that was provided from the organization. The data set consisted of historical data pertaining to compliance rates, types of HAIs acquired, and quantity of HAIs accumulated for each year. Figure 1 captures this dataset and consists of 3.5 years of data for analysis. The average $\mathrm{HH}$ compliancy for year 2017 was $80 \%$, which means that this year was considered noncompliant with CDC HH standards because $90 \%$ is the minimum expectation. As for HAIs, Clostridium difficile led the way each year, with CAUTI coming in second, CLABSI being third, and methicillin-resistant Staphylococcus aureus being last. 


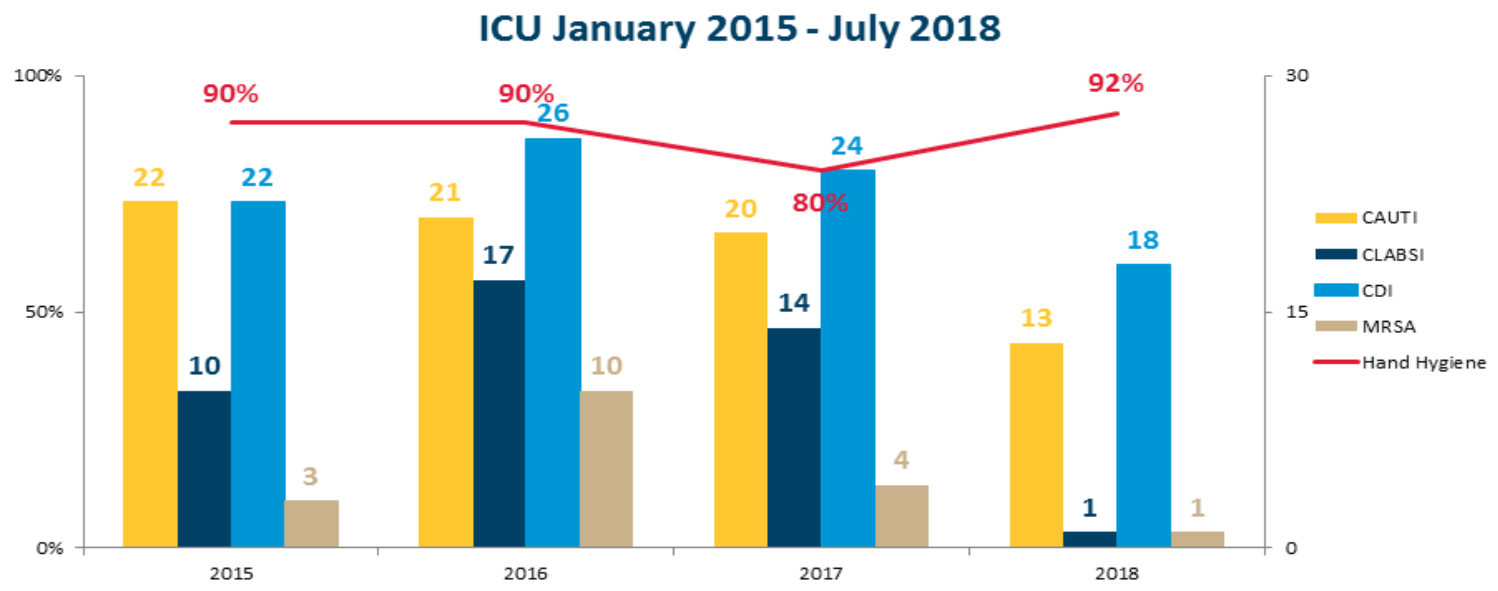

Figure 1. 3.5 Years of Hand-Hygiene Compliancy Data for the Intensive Care Unit (ICU). CAUTI= catheter-associated urinary tract infection; $C L A B S I=$ central-line-associated bloodstream infection; $C D I=$ Clostridium difficile; $M R S A=$ methicillin-resistant Staphylococcus aureus.

\section{Standardized Infection Ratio (SIR) for ICU}

The final secondary data that was requested from the organization to address RQ1 was the SIR. According to the CDC (2018), the SIR is a summary measure used to track HAIs at a national, state, or local level over time. The organization provided the SIRs for CAUTI and CLABSI. Figure 2 shows that the SIR for each year of CAUTIs was greater than the benchmark of 1.0, which, according to the $\mathrm{CDC}$, reflects that there is a need for stronger HAI prevention efforts.

ICU CAUTI 2015 - July 2018

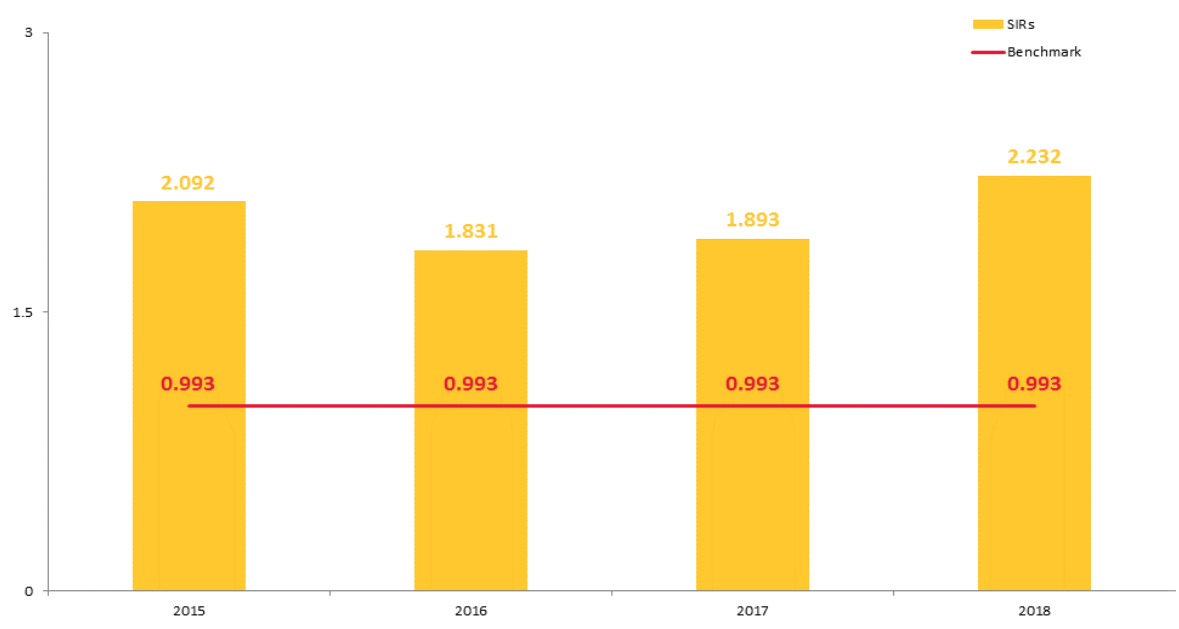

Figure 2. 3.5 Years of Standardized Infection Ratios (SIRs) for Catheter-Associated Urinary Tract Infection (CAUTI) in the Intensive Care Unit (ICU) 
Figure 3 shows that the SIR for each year of CLABSIs observed was also greater than the benchmark of 1.0, which reflects that there is a need for stronger HAI prevention efforts.

ICU CLABSI 2015 - July 2018

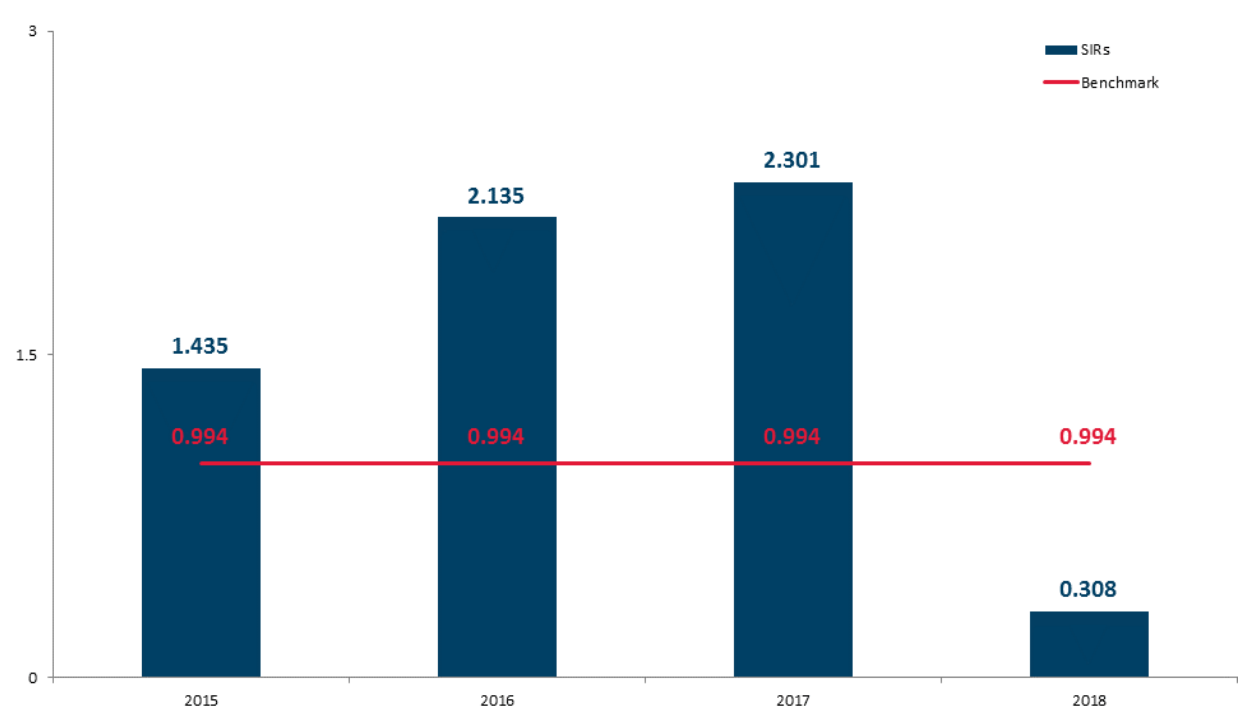

Figure 3. 3.5 Years of Standardized Infection Ratios (SIRs) for Central-Line-Associated Bloodstream Infection (CLABSI) in the Intensive Care Unit (ICU)

\section{Direct Observation Results to Address RQ1 (Excluded From RQ2 and RQ3)}

The researcher conducted direct observation on $35 \mathrm{HCWs}$ ( 15 doctors, 15 nurses, and five patient care technicians). The 15 physicians observed yielded an overall $\mathrm{HH}$ compliancy level of $77 \%$, the 15 nurses yielded compliancy level of $73 \%$, and the five physician care technicians yielded compliancy level of $90 \%$. An observation constituted of observing an entry and exit from the patient's room. For a side-by-side average compliance comparison for individuals, the patient care technicians were the only group meeting $\mathrm{HH}$ goal of $90 \%$. See Figure 4 for data. 
ICU Manual Obs.

Average Compliance

(By Individual)

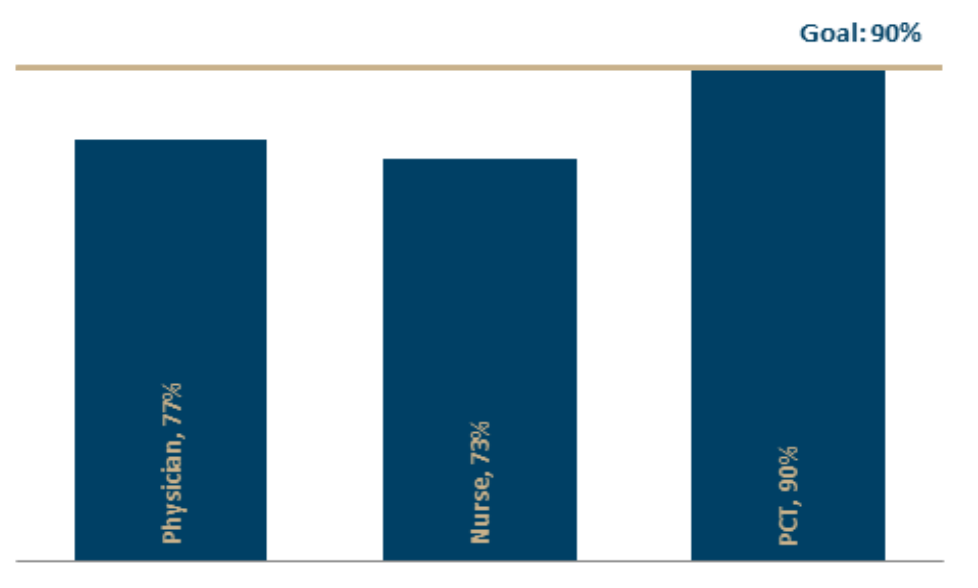

Figure 4. Average Compliance for Individuals. ICU = intensive care unit; Obs. = observation; PCT = patient care technician.

For RQ2, the only data collection method that was used to address was the questionnaire. The researcher compared the frequency of responses for 21 participants to identify any themes from the parent node, which was how leadership can motivate HCWs. The analysis of five questions was used in Table 2 to address RQ2.

\section{Table 2. Questionnaire to Address Research Question 2: How Leadership Can Motivate} Healthcare Workers (HCWs)

\begin{tabular}{|c|c|c|c|}
\hline Survey Question & Top Response & $n$ & $\%$ \\
\hline \multirow[t]{2}{*}{$\begin{array}{l}\text { 1. Steps leadership has taken or should take to } \\
\text { bolster HH }\end{array}$} & $\begin{array}{l}\text { Compliance reports and } \\
\text { feedback }\end{array}$ & 5 & $24 \%$ \\
\hline & Reminders-posted signs & 5 & $24 \%$ \\
\hline $\begin{array}{l}\text { 2. Leader qualities to drive change healthcare } \\
\text { organization }\end{array}$ & Lead by example & 8 & $38 \%$ \\
\hline \multirow{2}{*}{$\begin{array}{l}\text { 3. How leadership can motivate HCW'' HH } \\
\text { compliance }\end{array}$} & Hold others accountable & 6 & $29 \%$ \\
\hline & Material incentives & 6 & $29 \%$ \\
\hline $\begin{array}{l}\text { 4. Leadership role to meet and maintain } \mathrm{HH} \\
\text { compliance }\end{array}$ & Educate-remind-feedback & 7 & $33 \%$ \\
\hline $\begin{array}{l}\text { 5. Organizational culture meets and maintains } \\
\text { HH compliance }\end{array}$ & Changes needed & 14 & $67 \%$ \\
\hline 6. What to do if team members not practicing $\mathrm{HH}$ & Remind & 17 & $81 \%$ \\
\hline
\end{tabular}

Note. $N=21 . \mathrm{HH}=$ hand hygiene.

For RQ3, the only data collection method that was used to address was the questionnaire. The researcher compared the frequency of responses for 21 participants to identify any themes from the parent node, which was impact of technology. The analysis of five questions was used in Table 3 to address RQ3. 
Table 3. Questionnaire to Address Research Question 3: Impact of Technology

\begin{tabular}{llcc}
\hline Survey Question & \multicolumn{1}{c}{ Top Response } & $\boldsymbol{n}$ & $\mathbf{\%}$ \\
\hline 1. Opinion of RFID technology to monitor HH & Negative & 10 & $48 \%$ \\
& Malfunction-prone to error & 5 & $24 \%$ \\
& No-did not like & 5 & $24 \%$ \\
2. Other technologies to monitor HH & Unsure-none & 17 & $81 \%$ \\
3. Technology help or hinder HH & Help & 12 & $57 \%$ \\
& Hinder & 8 & $38 \%$ \\
4. Greatest impact technology can have HH & Positive & 11 & $52 \%$ \\
& Increased compliance & 6 & $29 \%$ \\
5. Additional feedback or concerns technology HH & Decrease infection rates & 4 & $19 \%$ \\
\hline
\end{tabular}

Note. $N=21 . \mathrm{RFID}=$ radio frequency identification; $\mathrm{HH}=$ hand hygiene.

\section{Examination of RQs}

The discovery of data through the secondary data from the organization, the direct observation conducted by the researcher, and the questionnaire responses from HCWs provided rich data to analyze the findings from the three RQs, which were used to help frame the study.

\section{RQ1}

The first set of findings for RQ1 was based on the results from the questionnaire. The questionnaire was used to address all three RQs. First, HCWs accepted direct observation as the best practice used to monitor $\mathrm{HH}$ and cited being too busy as the major factor for not practicing $\mathrm{HH}$. Secondly, HCWs also cited empty or broken foam dispensers as a factor for hindering their ability to practice $\mathrm{HH}$, which was beyond their behavior. The last key finding from the questionnaire used for RQ1 was that HCWs felt empowered to remind team members to practice $\mathrm{HH}$ if they noticed them being noncompliant.

The overall interpretation from the above findings was that the direct observation method was received as the favorable method among $\mathrm{HCW}$ s to monitor $\mathrm{HH}$, and that a busy environment and nonworking foam dispenser's results in poor $\mathrm{HH}$ rates. However, even though HCWs viewed direct observation as best practice, this practice produced unacceptable compliance rates for physicians and nurses. After employing triangulation for the three data collection methods, the final analysis for RQ1 was that the gold standard used to monitor $\mathrm{HH}$, direct observation, has produced unfavorable compliance rates and HAI metrics for the ICU.

\section{RQ2}

For RQ2, HCWs believed that to motivate peers to practice HH, leadership must hold HCWs accountable. In addition to holding others accountable, HCWs cited receiving incentives as a motivational factor. The interpretation for this finding was that some HCWs believed that they were doing the right thing, but if the organization does not enforce accountability measures to all, then some see this problem as ongoing. To validate this observation, $67 \%$ of $\mathrm{HCWs}$ concluded that a change in the organizational culture was needed to help meet and maintain HH compliancy requirements. In comparison, $24 \%$ cited that they valued current cultural attributes, namely, that the organization understands the importance of HH. This means that HCWs do see this as a serious problem and would like to see different strategies employed to help achieve goals of meeting $\mathrm{HH}$ standards. As a starting point, $38 \%$ of $\mathrm{HCW}$ s believed that to drive change within the organization, leaders must lead by example. 


\section{RQ3}

For RQ3, 48\% HCWs did not have a favorable view of using RFID technology to monitor $\mathrm{HH}$, but $52 \%$ felt positive about using technology in general, with $19 \%$ believing that technology can have a great impact to decrease infections rates and $29 \%$ believing it can increase compliance. The interpretation for this finding was that HCWs believed that technology can be used as a support tool for monitoring $\mathrm{HH}$, but the proper application and its usefulness needs to be communicated to the healthcare community. To confirm this observation, when HCWs were asked to identify any other technology besides RFID to monitor $\mathrm{HH}, 81 \%$ could not make any other references. In closing, the overall findings resulting from the three RQs provided rich data for analysis and recommendations for healthcare leadership.

\section{Recommendation for Healthcare Leadership}

As highlighted by Cain et al. (2018), a proficient and engaged nursing staff is key to achieving quality and safety outcomes, which are essential for an organization to remain competitive. Nurses must be adept at clinical reasoning, navigate patient care situations in coordination with an interprofessional team, be skilled communicators, and be proficient in technology. Nurses who are successful in these areas will approach difficult tasks as challenges, thrive in the face of situational complexities, and be risk takers when innovation is sought. Each of the above characteristics related to a registered nursing workforce demonstrates a commitment to unit goals and to the organization and will have greater tolerance during episodic times of rapid change.

With that said, the researcher chose to focus this study on the ICU because studies have shown that out of all the care units within a hospital, the ICU has shown to have the highest cases of HAIs (Radhakrishna et al., 2015). What is more, Martins Pereira et al. (2016) noted that compared to palliative care, working in ICUs more than doubled the likelihood of exhibiting burn out for HCWs. Therefore, given the myriad of challenges that the nursing workforce is facing, the dilemma presented to leadership is the question of what leadership style should be selected for promoting workplace engagement and reducing burnout among nurses while simultaneously focusing on reducing HAIs. Although there are many identified styles of leadership that are available for healthcare leaders to choose from (e.g., transformational, transactional, autocratic, laissez-faire, task-oriented, and relationship-oriented leadership), the researchers are recommending a new leadership theory to be used.

This new leadership theory is called servant leadership. For the past 4 decades, servant leadership has evolved as a reputable leadership theory and construct. Servant leadership offers a multidimensional leadership theory that encompasses all aspects of leadership, including ethical, relational, and outcome-based dimensions. Servant leadership is like, but also different from, current leadership theories and proposes a more meaningful way of leadership to ensure sustainable results for individuals, organizations, and societies. One of the core tenets of servant leadership theory is that servant leaders instill in followers a desire to serve others, which is what healthcare is all about (Lacroix \& Verdorfer, 2017).

\section{Discussion and Conclusion}

\section{Summary and Implications}

On the whole, handwashing has been shown to be the single most effective way to combat disease and infection transmission (CDC, 2012). In other words, when proper handwashing is not performed, such as washing with soap and water or using alcohol-based rubs, bacteria are more likely to spread when HCWs care for patients. Despite this known revelation, compliance levels among HCWs remain variable and disappointingly low (Chassin et al., 2015). Studies have found a range of factors 
such as lack of accountability, HCW being distracted, HCW being too busy, or simply employee burnout as noted reasons for not practicing proper HH (Martins Pereira et al., 2016).

According to Klevens et al. (2007), the result of not properly practicing proper $\mathrm{HH}$ is directly linked to producing the four most common HAIs in hospitals, which are CAUTI, surgical-site infections, VAP, and CLABSI. To assist HCWs to minimize the spread of HAIs and to meet CDC HH standards, there are notable best practices that are used within hospitals. Practices such as direct observation, which has been described as the gold standard approach to $\mathrm{HH}$, product volume measurement, and ECM have all been documented as best practices. Yet, the spread of HAIs continues to add financial burdens to healthcare facilities, increase the death rate, and add tremendous emotional stress for patients and their families.

Consequently, healthcare leaders continue to struggle with finding the optimal solution to address these problems. A potential solution that has been hailed as the savior to help meet CDC HH standards is RFID sensor technology. RFID technology uses sensors to help remind HCWs to perform $\mathrm{HH}$ as required before coming in contact with patients. In spite of its claim to help HCWs meet CDC HH standards, noted barriers were reviewed to give more insight into the complexities within healthcare when adopting technologies become the focus. Nevertheless, the final analysis is that as the healthcare industry continues to adapt to an ever-changing environment, the two goals that needs to be constant is to treat patient's illnesses and to keep them safe.

\section{Recommendations for Future Research}

Future research is necessary around other technologies that promote reducing HAIs besides RFID, such as video cameras. In addition, because the research was limited to the sample population described above, future researchers should include doctors, nurses, and patient care technicians in private teaching hospitals within other U.S. metropolitan cities. A third recommendation would be to expand the research to compare ICU HCWs to other care units within the hospital to identify systematic barriers around meeting HH compliancy. For this study, the researcher should employ a quantitative correlational design utilizing statistical methods, which would add rigor and reliability and will thus make the conclusions more generalizable. The final suggestion for future research would be to explore a qualitative case study within the ICU and explicitly ask respondents whether and to what degree they desire a leader who engages in servant leadership behaviors as a strategy toward reducing HAIs.

\section{References}

Al Salman, J. M., Hani, S., de Marcellis-Warin, N., \& Isa, S. F. (2015). Effectiveness of an electronic hand-hygiene monitoring system on healthcare workers' compliance to guidelines. Journal of Infection and Public Health, 8, 117-126. https://doi.org/10.1016/j.jiph.2014.07.019

Baker, M., \& Wilson, N. (2007). Chicken meat is clearly the most important source of human campylobacter infection in New Zealand. New Zealand Journal of Medical Laboratory Science, 61, 44-47.

Baslyman, M., Rezaee, R., Amyot, D., Mouttham, A., Chreyh, R., Geiger, G., . . Sader, S. (2015). Real-time and location-based hand hygiene monitoring and notification: Proof-of-concept system and experimentation. [Retrieved from ProQuest Database]. Personal and Ubiquitous Computing, 19, 667-688. https://doi.org/10.1007/s00779-015-0855-y

Boyce, J. M. (2017). Electronic monitoring in combination with direct observation as a means to significantly improve hand hygiene compliance. American Journal of Infection Control, 45, 528-535. https://doi.org/10.1016/j.ajic.2016.11.029 
Cain, L. B., Cronin, S. N., Nelson, D., Meredith, D. A., Newman, K. P., \& Rudolf, S. (2018). A tool to identify key behaviors and attributes of high-performing nurses. The Journal of Nursing Administration, 48, 197-202. https://doi.org/10.1097/NNA.0000000000000599

Carr, A. S. (2015). An examination of the adoption of RFID technology in healthcare organizations. Organization Development Journal, 33, 81-102.

Centers for Disease Control and Prevention (CDC). (2012). Eliminating healthcare associated infections: State policy options. Retrieved from https://www.cdc.gov/hai/pdfs/toolkits/toolkithai-policy-final_01-2012.pdf

Centers for Disease Control and Prevention (CDC). (2013). Resistance threats in the United States. Retrieved from https://www.cdc.gov/drugresistance/pdf/ar-threats-2013-508.pdf

Centers for Diseases and Control Prevention (CDC). (2017a). HAI data and statistics. Retrieved from https://www.cdc.gov/hai/surveillance/index.html

Centers for Diseases Control and Prevention (CDC). (2017b). Our story: Our history. Retrieved from https://www.cdc.gov/about/history/ourstory.htm

Centers for Disease Control and Prevention (CDC). (2017c). Types of healthcare-associated infections. Retrieved from https://www.cdc.gov/hai/organisms/organisms.html

Center for Diseases Control and Prevention (CDC). (2018). The NHSN standardized infection ratio (SIR). Retrieved from https://www.cdc.gov/nhsn/pdfs/ps-analysis-resources/nhsn-sir-guide.pdf

Chassin, M. R., Mayer, C., \& Nether, K. (2015). Improving hand hygiene at eight hospitals in the United States by targeting specific causes of noncompliance. Joint Commission Journal on Quality and Patient Safety, 41, 4-12. https://doi.org/10.1016/S1553-7250(15)41002-5

Creswell, J. W. (2005). Research Design: Qualitative, quantitative, and mixed methods approaches. Los Angeles, CA: Sage Publications.

Creswell, J. W. (2014). Research design: Qualitative, quantitative and mixed methods Approaches (4th ed.). Thousand Oaks, CA: Sage Publications.

Ellingson, K., Haas, J. P., Aiello, A. E., Kusek, L., Maragakis, L. L., Olmsted, R. N., . . Yokoe, D. S., \& the Society for Healthcare Epidemiology of America (SHEA). (2014). Strategies to prevent healthcare-associated infections through hand hygiene. Infection Control and Hospital Epidemiology, 35, 937-960. https://doi.org/10.1086/651677

Elo, S., Kääriäinen, M., Kanste, O., Pölkki, T., Utriainen, K., \& Kyngäs, H. (2014). Qualitative content analysis: A focus on trustworthiness. SAGE Open, 4, 110. https://doi.org/10.1177/2158244014522633"

Erasmus, V., Daha, T. J., Brug, H., Richardus, J. H., Behrendt, M. D., Vos, M. C., \& van Beeck, E. F. (2010). Systematic review of studies on compliance with hand hygiene guidelines in hospital care. Infection Control and Hospital Epidemiology, 31, 283294. https://doi.org/10.1086/650451

Glogowska, M. (2011). Paradigms, pragmatism and possibilities: Mixed-methods research in speech and language therapy. International Journal of Language \& Communication Disorders, 46, 251-260. https://doi.org/10.3109/136822.2010.507614

Gould, D. J., Creedon, S., Jeanes, A., Drey, N. S., Chudleigh, J., \& Moralejo, D. (2017). Impact of observing hand hygiene in practice and research: A methodological reconsideration. The Journal of Hospital Infection, 95, 169-174. https://doi.org/10.1016/j.jhin.2016.08.008

Hagel, S., Reischke, J., Kesselmeier, M., Winning, J., Gastmeier, P., Brunkhorst, F. M., . . Pletz, M. W. (2015). Quantifying the Hawthorne effect in hand hygiene compliance through comparing 
direct observation with automated hand hygiene monitoring. Infection Control and Hospital Epidemiology, 36(8), 957-962. https://doi.org/10.1017/ice.2015.93

Hong, T. S., Bush, E. C., Hauenstein, M. F., Lafontant, A., Li, C., Wanderer, J. P., \& Ehrenfeld, J. M. (2015). A hand hygiene compliance check system: Brief communication on a system to improve hand hygiene compliance in hospitals and reduce infection. Journal of Medical Systems, 39, 69. https://doi.org/10.1007/s10916-015-0253-z

Kash, B. A., Spaulding, A., Johnson, C. E., Gamm, L., \& Hulefeld, M. F. (2014). Success factors for strategic change initiatives: A qualitative study of healthcare administrators' perspectives. Journal of Healthcare Management, 59, 6581. https://doi.org/10.1097/00115514-201401000-00011

Klevens, R. M., Edwards, J. R., Richards, C. L., Jr., Horan, T. C., Gaynes, R. P., Pollock, D. A., \& Cardo, D. M. (2007). Estimating health care-associated infections and deaths in U.S. hospitals, 2002. Public Health Reports, 122, 160166. https://doi.org/10.1177/003335490712200205

Lacroix, M., \& Verdorfer, A. P. (2017). Can servant leaders fuel the leadership fire? The relationship between servant leadership and followers' leadership avoidance. Administrative Sciences, 7, 1-11. https://doi.org/10.3390/admsci7010006

Marques, R., Gregório, J., Pinheiro, F., Póvoa, P., da Silva, M. M., \& Lapão, L. V. (2017). How can information systems provide support to nurses' hand hygiene performance? Using gamification and indoor location to improve hand hygiene awareness and reduce hospital infections. BMC Medical Informatics and Decision Making, 17, 15. https://doi.org/10.1186/s12911-017-0410-z

McGuckin, M., \& Govednik, J. (2015). A review of electronic hand hygiene monitoring: Considerations for hospital management in data collection, healthcare worker supervision, and patient perception/practitioner application. Journal of Healthcare Management, 60, 348361. https://doi.org/10.1097/00115514-201509000-00009

Neo, J. R., Sagha-Zadeh, R., Vielemeyer, O., \& Franklin, E. (2016). Evidence-based practices to increase hand hygiene compliance in health care facilities: An integrated review. American Journal of Infection Control, 44, 691-704. https://doi.org/10.1016/j.ajic.2015.11.034

Pandey, S. C., \& Patnaik, S. (2014). Establishing reliability and validity in qualitative inquiry: A critical examination. Journal of Development and Management Studies, 12, 5743-5753.

Martins Pereira, S., Teixeira, C. M., Carvalho, A. S., \& Hernández-Marrero, P. (2016). Compared to palliative care, working in intensive care more than doubles the chances of burnout: Results from a nationwide comparative study. PLoS One, 11, e0162340. Retrieved from https://doi.org/10.1371/journal.pone.0162340

Radhakrishna, K., Waghmare, A., Ekstrand, M., Raj, T., Selvam, S., Sreerama, S. M., \& Sampath, S. (2015). Real-time feedback for improving compliance to hand sanitization among healthcare workers in an open layout ICU using radiofrequency identification. Journal of Medical Systems, 39, 68. https://doi.org/10.1007/s10916-015-0251-1

Simon, M., \& Goes, J. (2013). Dissertation and scholarly research: A practical guide to start and complete your dissertation, thesis, or formal research project: Recipes for success. CreateSpace Independent Publishing Platform.

van Dijk, M. D., Mulder, S. A., Erasmus, V., van Beeck, A. H. E., Vermeeren, J. M. J. J., Liu, X., . . . Vos, M. C. (2019). A multimodal regional intervention strategy framed as friendly competition to improve hand hygiene compliance. Infection Control and Hospital Epidemiology, 40, 187-193. https://doi.org/10.1017/ice.2018.261 
World Health Organization (WHO). (2011). Report on the burden of endemic health care-associated infection worldwide. Retrieved from http://apps.who.int/iris/bitstream/10665/80135/1/9789241501507_eng.pdf?ua=1

Yin, R. K. (2009). Case study research design and methods (4th ed.). Thousand Oaks, CA: Sage Publication.

The International Journal of Applied Management and Technology (IJAMT), sponsored by Walden University's School of Management, is a peer-reviewed, online journal that addresses contemporary national and international issues related to management and technology. The objectives of the IJAMT are to: (a) encourage collaborative and multi-disciplinary examinations of important issues in business and technology management, and (B) engage scholars and scholar-practitioners in a dynamic and important dialogue.

Walden University Publishing: http://www.publishing.waldenu.edu 